Pacific Journal of Mathematics

A GENERAL SOLUTION OF TONELLI'S PROBLEM OF THE 


\title{
A GENERAL SOLUTION OF TONELLI'S PROBLEM OF THE CALCULUS OF VARIATIONS
}

\author{
LIONELLO A. LOMBARDI
}

Summary. The alpha-asymptotical property is defined for integrals depending upon any number $m$ of surfaces of any dimension in nonparametric form. The existence of an absolute minimum of any alphaasymptotical and lower semicontinuous integral in any regular class of varieties which is closed with respect to the m-uniform (Tchebyshev) metric is proved.

1. Definitions. Let $D_{i}(i=1,2, \cdots, m)$ be a closed bounded set of the $n$-dimensional Euclidean space of the variable vector $x_{i} \equiv x_{i}^{j}(j=$ $1,2, \cdots, n)$, bounded by surfaces which are absolutely continuous in the sense of Tonelli $[14,16,17]$, without multiple points, and let $D$ be the cartesian product $\Pi_{1^{i}}^{m} D_{i}$. Let $y \equiv y_{i}(i=1,2, \cdots, m)$ denote a vertical $m$-vector, and let $p$ denote an $m x n$ matrix, whose row-vectors are $p_{i} \equiv$ $p_{i}^{j}(j=1,2, \cdots, n)$. Let $x$ be the mxn matrix whose row-vectors are $x_{i}$ and $\phi[x, y, p]$ a real-valued function, defined for $x_{i} \in D_{i}(i=1,2, \cdots, m)$ and for any $y$ and $p$, which is continuous with all its derivatives of the types

$$
\frac{\partial \phi[x, y, p]}{\partial p_{r}^{s}} \text { and } \frac{\partial^{2} \phi[x, y, p]}{\partial p_{r}^{s} \partial p_{r}^{t}} \quad(r=1, \cdots, m ; s, t=1 \cdots n) .
$$

Let $q \leqq m$ be a positive integer and let $U_{q}$ denote a set of $q$ distinct positive integers out of the first $m$; let $\zeta$ be an index ranging over $U_{q}$, and let $\mu(\zeta)$ be a mapping of $U_{q}$ into the set of the first $n$ integers. It will be assumed throughout, that for every $q$, every $U_{q}$ and every $\mu(\zeta)$, all the partial derivatives

$$
\frac{\partial^{2 q} \phi[x, y, p]}{\prod_{\zeta=1}^{q} \partial x_{\zeta}^{\mu(\zeta)} \partial p_{\zeta}^{\mu(\zeta)}}
$$

exist and are continuous for every $x \in D$ and for every $y$ and $p$.

Let $T$ be a real positive number. Let $y(x) \equiv y_{i}\left(x_{i}\right)(i=1,2, \cdots, m)$ denote a vector-valued function of the matrix $x$, such that each $y_{i}\left(x_{i}\right)$ is a function with values in $[-T, T]$, which only depends upon the row

Received May 26, 1961. The preparation of this paper was sponsored by the Office of Naval Research. Reproduction in whole or in part is permitted for any purpose of the United States Government. Now with the School of Industrial Management at the Massachusetts Institute of Technology.

The author wishes also to express his thanks to Gerald W. Kimble for valuable help in preparing the manuscript. 
vector $x_{i}$. We assume that each $y_{i}\left(x_{i}\right)$ is absolutely continuous, in the sense of Tonelli [17]; we shall call variety $V$ the set of $m$ surfaces represented by $y(x)$, and the functions $y_{i}\left(x_{i}\right)$ will be called components of $y(x)$.

Let

$$
p_{i}^{j}(x) \equiv \frac{\partial y_{i}\left(x_{i}\right)}{\partial x_{i}^{j}} \quad(i=1,2, \cdots, m ; j=1,2, \cdots, n)
$$

and

$$
d x \equiv \prod_{i=1}^{m} d x_{i} \equiv \prod_{i=1}^{m} \prod_{j=1}^{n} d x_{i}^{j}
$$

The $m x n$ integral

$$
I_{V} \equiv \int_{D} \phi[x, y(x), p(x)] d x
$$

is called a variety integral in nonparametric form; all the varieties $V$ where $I$ exists and is finite are called ordinary.

Let $\bar{p} \equiv \bar{p}_{i} \equiv \bar{p}_{i}^{j}$ denote another variable in the space of the matrix $p$, $\bar{y} \equiv \bar{y}_{i}$ another variable in the space of the vector $y, \quad V \equiv \bar{y}(x) \equiv \bar{y}_{i}\left(x_{i}\right)$ another variety $V$; let

$$
\bar{p}_{i}^{j}\left(x_{i}\right)=\frac{\partial \bar{y}_{i}\left(x_{i}\right)}{\partial x_{i}^{j}}
$$

The distance $\rho(V, \bar{V})$ between $V$ and $\bar{V}$ is defined by the formula

$$
\rho(V, \bar{V})=\sup _{x, i}\left|y_{i}(x)-\bar{y}_{i}(x)\right| \text {. }
$$

In the sequel (whenever not otherwise specified) all of the topological properties of spaces and families of varieties $V$ will refer to this m-uniform (Tchebychev) metric.

Let us assume by convention that if $\eta$ is a variable integer ranging over a set $S$ and $\left\{\alpha_{\eta}\right\}$ is a sequence of numbers, then

$$
\prod_{\eta \in S} \alpha_{\eta}=0 \text {, whenever } S \text { is empty . }
$$

Let $L[x, y, p, \bar{p}]$ denote a polynomial in the indeterminates

$$
\left[\bar{p}_{i}^{(j)}-p_{i}^{(j)}\right]
$$

of degree not exceeding 1 in any of the vectors $\left[\bar{p}_{i}-p_{i}\right]$, whose coefficients $W_{U_{q}, \mu}(x, y, p)$ are functions of $(x, y, p)$ which are continuous together with all their derivatives of the form

$$
\frac{\partial^{q} W_{U_{q}, \mu}(x, y, p)}{\prod_{\zeta \in U_{q}} x_{\zeta}^{\mu(\zeta)}}
$$


$L[x, y, p, \bar{p}]$ may be written in the form

$$
\sum_{q-1} \sum_{U_{q}} \sum_{\mu} W_{U_{q}, \mu}(x, y, p) \prod_{J_{q}}\left[\bar{p}_{\zeta}^{\mu(\zeta)}-p_{\zeta}^{\mu(\zeta)}\right]
$$

The generalized Weierstrass function of $I_{V}$ with respect to $L[x, y, p, \bar{p}]$ is defined by the formula

$$
\mathscr{E}_{L}(x, y, p, \bar{p})=\phi[x, y, \bar{p}]-L[x, y, p, \bar{p}] .
$$

The integral $I_{V}=\int \phi[x, y(x), p(x)] d x$ is said to be positively quasiregular with respect to $L$ (abbreviation: $L P Q R$ ) if both the relations

$$
\begin{aligned}
& \mathscr{E}_{L}[x, y, p, p]=0 \\
& \mathscr{E}_{L}[x, y, p, \bar{p}] \geqq 0
\end{aligned}
$$

hold for every $x \in D$ and for every $y, p, \bar{p}$.

We say that $I_{V}$ is positively quasi-regular (abbreviation: $P Q R$ ) if there exists at least one function $L[x, y, p, \bar{p}]$ such that $I_{V}$ is $L P Q R$.

REMARK 1.1. If $I_{V}$ is $P Q R$, then its value at every nonordinary variety is $+\infty$.

Suppose now that $I_{V} \equiv \int_{D} \phi[x, y(x), p(x)] d x$ is $P Q R$ and let $L[x, y, p, \bar{p}]$ be one of the functions such that $I_{V}$ is $L P Q R$.

The function

$$
\bar{\phi}[x, y, p]=\mathscr{E}_{L}[x, y, \Omega, p],
$$

where $\Omega$ is an $m x n$ matrix whose elements are all 0 , is never negative. Furthermore,

$$
\bar{I}_{\nabla}=\int_{D} \bar{\phi}[x, y(x), p(x)] d x
$$

is $L P Q R$, where

$$
\bar{L}[x, y, p, \bar{p}]=L[x, y, p, \bar{p}]-L[x, y, \Omega, \bar{p}] .
$$

By (1.6), the equation

$$
\bar{\phi}[x, y, \Omega]=0
$$

holds for every $X \in D$ and every $y$.

Let $R$ denote a positive real number and let $\varphi^{R}[x, y, p]$ denote a function such that the following conditions are satisfied:

I. $\varphi^{R}[x, y, p]$ is continuous with all its partial derivatives of any of the forms 


$$
\frac{\partial \varphi^{R}[x, y, p]}{\partial p_{r}^{s}}, \frac{\partial^{2} \varphi^{R}[x, y, p]}{\partial p_{r}^{s} \partial p_{r}^{t}}, \frac{\partial^{2 q} \varphi^{R}[x, y, p]}{\prod_{1 \zeta}^{q} \partial x_{\zeta}^{\mu(\zeta)} \partial p_{\zeta}^{\mu(\zeta)}}
$$

II. The integral

$$
Y_{V}=\int_{D} \varphi^{R}[x, y(x), p(x)] d x
$$

is $P Q R$.

III. The relation

$$
0 \leqq \phi^{R}[x, y, p] \leqq \bar{\phi}[x, y, p]
$$

holds for every $y, p$ and for every $x \in D$; furthermore,

$$
\varphi^{R}[x, y, p]=\bar{\phi}[x, y, p], \quad \text { whenever } \sum_{i=1}^{m} \sum_{j=1}^{n}\left(p_{i}^{j}\right)^{2} \leqq R .
$$

IV. There exists at least one function $\Lambda[x, y, p, \bar{p}]$, such that $Y_{V}$ is $A P Q R$, and such that there exists a number $Q$, for which the following condition is satisfied:

Let $q, U_{q}, \zeta, \mu(\zeta)$ be defined as they were above; let $\bar{U}_{q}$ denote the complement of $U_{q}$ with respect to the set of the first $m$ positive integers, and let $\bar{\zeta}$ be an index ranging over $\bar{U}_{q}$. Then the inequality

$$
\left|W_{U q, \mu}^{R}[x, y, p]\right|<Q\left(1+\prod_{\bar{\zeta} \in \bar{U}_{q}}\left|p_{\bar{\zeta}}^{\mu}(\bar{\zeta})\right|\right)
$$

where $W_{U_{q}, \mu}^{R}[x, y, p]$ denotes the coefficient of the element

$$
\prod_{\zeta \in U_{q}}\left[\bar{p}_{\zeta}^{\mu(\zeta)}-p_{\zeta}^{\mu(\zeta)}\right]
$$

of the function $\Lambda[x, y, p, \bar{p}]$, holds for every $q, U_{q}, p$, for every $x \in D$, for every $\mu$ and for every $y$, such that

$$
\left|y_{i}\right| \leqq T, \quad(i=1,2, \cdots, n) .
$$

The integral $I_{V}$ is said to be asymptotically evaluable $[8,12]$ (abbreviation: AE) when there exists a function $L[x, y, p, \bar{p}]$ such that $I_{V}$ is $L P Q R$ and such that for every positive $R$ there exists a function $\varphi^{R}[x, y, p]$ as described above. By using continuity lemmas proved in [11], in [12] this author proved the following:

Semicontinuity Theorem 1.1. An integral $I_{V}$ which is $P Q R$ and $A E$ is lower semicontinuous at every variety $V$, ordinary or not. (See also $[8,13,14])$.

REMARK 1.2. If $V$ is a nonordinary variety and $I_{V}$ is $P Q R$, the 
lower semicontinuity of $I_{V}$ at $V$ denotes the fact that if $\left\{V_{i}\right\}$ is any sequence of varieties such that

$$
\lim _{i \rightarrow \infty} V_{i}=V \text {, }
$$

then

$$
\lim _{i \rightarrow \infty} I_{V_{i}}=+\infty
$$

Definition. We shall say that an integral $I_{V} \equiv \int_{D} \phi[x, y, p] d x$ is alpha-asymptotic if there exist two real numbers $\alpha>0$ and $Y>1$ such that

$$
\phi(x, y, p)>\prod_{i=1}^{m} \sum_{j=1}\left|p_{j}^{i}\right|^{n+\infty}
$$

whenever

$$
\prod_{i=1}^{m} \sum_{j=1}\left|p_{i}^{j}\right|>Y
$$

and if $\phi[x, y, p]$ is bounded below by a number $H$.

We shall say that a set $F$ of varieties has the property $A$ if there exist two real numbers $\beta>0$ and $M$ such that for each $V \equiv y(x) \in F$,

$$
\int_{D} \prod_{i=1}^{m} \sum_{j=1}^{n}\left|p_{i}^{j}\left(x_{i}\right)\right|^{n+\beta} d x \leqq M .
$$

We shall say that $F$ has the property $B$ if there exist two real numbers $\gamma>0$ and $N$ such that, for each $V \equiv y(x) \in F$,

$$
\int_{D} \sum_{j=1}^{n}\left|p_{i}^{j}\left(x_{i}\right)\right|^{n+\gamma} \leqq N, \quad(i+1,2, \cdots, m) .
$$

Definition. A set $\mathscr{F}$ of varieties is called a regular class if each set $F \subset \mathscr{F}$ which has property $A$ has also property $B$.

REMARK. For $m=1$, every set of varieties is a regular class. If all the $m$ pointsets $D_{i}$ and the $m$ components $y_{i}\left(x_{i}\right)$ of a variety $V$ are identical to each other, we shall say that $V$ is symmetric. If $\mathscr{F}$ consists only of symmetric varieties it is clearly a regular class: however, sets of symmetric varieties are only a very special case of regular classes. Clarifying examples of regular classes and regularity criteria were given in [9] for the case $m=2, n=1$ (Fubini-Tonelli integrals).

DeFinition. A set $\mathscr{F}$ of ordinary varieties which contains all the ordinary varieties belonging to the boundary of $\mathscr{F}$ is called a complete class. 
ExAmples. Any closed set of varieties is a complete class. Both the set of all varieties and the set of all ordinary varieties are also complete classes.

2. The existence of the minimum. (a) The Problem of Tonelli consists of giving conditions on $\phi[x, y, p]$ which yield the existence of the minimum of $I_{V}$ in any regular complete class $\mathscr{F}$ of varieties.

Only conditions on $\phi[x, y, p]$ are allowed. In fact, several types of conditions on $\mathscr{F}$ (such as uniform bounds of the m-gradients $p(x)$ of the varieties of $\mathscr{F}$ or of their integrals) would easily yield compactness of $\mathscr{F}$ itself and a fortiori the existence of the minimum. However, no such condition is consistent with the physical phenomena that can be described mathematically as minima of integrals of the calculus of variation or with the use of solutions of Tonelli's problem as tools for investigating the existence of solutions of partial differential and integrodifferential equations [5, 18]. Our purpose in this study is to discuss the existence of a minimum of $I_{V}$ in classes $\mathscr{F}$ of varieties which are not a priori compact, imposing conditions only on $\phi[x, y, p]$.

(b) LEMMA 2.b.1. If $I_{V}$ is alpha-asymptotic and bounded above on a set $F$ of varieties, then $F$ has property $A$.

Let $V \equiv y(x) \in F$, and let $K$ be the upper bound of $I_{V}$ over $F$. Let $D_{1}$ denote the set of points $x$ of $D$ such that

$$
\prod_{i=1}^{m} \sum_{j=1}^{n}\left|p_{i}^{j}\left(x_{i}\right)\right| \leqq Y
$$

and $D_{2}$ the complement of $D_{1}$ with respect to $D$. Then

$$
\begin{aligned}
& \int_{D} \prod_{i=1}^{m} \sum_{j=1}^{n}\left|p_{i}^{j}\left(x_{i}\right)\right|^{n+\alpha} d x \leqq \int_{D_{1}} \prod_{i=1}^{m} \sum_{j=1}^{n}\left|p_{i}^{j}\left(x_{i}\right)\right|^{n+\alpha} d x \\
& \quad+\int_{D_{2}} \phi[x, y(x), p(x)] d x \leqq\left(Y^{n+\alpha}+|H|\right) \mu(D)+K
\end{aligned}
$$

where $\mu(D)$ denotes the measure of $D$.

The lemma is then proved simply by posing

$$
M \equiv\left(Y^{n+\infty}+|H|\right) \mu(D)+K \text { and } \beta \equiv \alpha .
$$

Let now $\left\{V_{s}^{(r)}\right\}(s=0,1,2, \cdots, m)$ denote sequences $\left(V_{s}^{(1)}, V_{s}^{(2)}, V_{s}^{(3)}, \cdots\right)$ of varieties and $y_{i, s}^{(r)}\left(x_{i}\right)$ the $i$ th component of $V_{s}^{(r)}$. Let us prove the following

Compactness Theorem 2.b.2. If $I_{V}$ is alpha-asymptotic and bounded above on $\left\{V_{0}^{(r)}\right\}$ and $\left\{V_{0}^{(r)}\right\}$ is contained in a regular class $\mathscr{F}$, then there exists a variety $V^{(\infty)} \equiv y_{i}^{(\infty)}(i=1,2, \cdots, m)$ of accumulation for 
$\left\{V_{0}^{(r)}\right\}$.

For Lemma 2.b.1 $\left\{V_{0}^{(r)}\right\}$ has the property $A$; since $\left\{V_{0}^{(r)}\right\} \subset \mathscr{F}$ and $\mathscr{F}$ is a regular class, $\left\{V_{0}^{(r)}\right\}$ has also property $B$. Consequently, for each $i(i=1,2, \cdots, m)$ the $i$ th components $y_{i, i-1}^{(r)}\left(x_{i}\right)$ of the varieties $V_{i-1}^{(r)}$ are equiabsolutely continuous functions of the $n$ variables $x_{i}^{j}$, and form a compact set with respect to the uniform (Tchebychev) metric, i.e., there is a sequence $\left\{V_{i}^{(r)}\right\} \subset\left\{V_{i-1}^{(r)}\right\}$ and an absolutely continuous function $y_{i}^{(\infty)}\left(x_{i}\right)$ such that for each $\varepsilon>0$ there is an $\bar{r}$ such that

$$
\left|y_{i}^{(\infty)}\left(x_{i}\right)-y_{i}^{(r)}\left(x_{i}\right)\right|<\varepsilon
$$

whenever $x_{i} \in D_{i}$ and $r<\bar{r}$. Consequently, in the metric of the space of varieties, the variety $V^{(\infty)} \equiv y_{i}^{(\infty)}(i=1,2, \cdots, m)$ is a variety of accumulation for $\left\{V_{m}^{(r)}\right\}$ and, since $\left\{V_{m}^{(r)}\right\} \subset\left\{V_{0}^{(r)}\right\}$, also of $\left\{V_{0}^{(r)}\right\}$. The theorem is thus proved.

We are now in a position to prove the following

THEOREM 2.b.3 of Existence of the Minimum: If the integral $I_{V}$ is positively quasi-regular, asymptotically evaluable and alpha asymptotic, then it has an absolute minimum in every regular complete class $\mathscr{F}$ of ordinary varieties.

Let $i$ denote the lower bound of $I_{V}$ over $\mathscr{F}$. Let us show that $i$ is finite: indeed, if it were that

$$
i=-\infty
$$

there would exist a sequence $\left\{Y^{(r)}\right\} \subset \mathscr{F}$ of varieties such that

$$
I_{V}(r)<-r
$$

by the compactness Theorem 2.b.2 there would exist a variety $V^{(\infty)}$ of accumulation for $\left\{V^{(\infty)}\right\}$. Since $I_{V}$ is positively quasi-regular by hypothesis, $I_{V}^{\infty}$ exists, and

$$
I_{V(\infty)}>-\infty
$$

(see Remark 1.1).

But by the semicontinuity Theorem 1.1, $I_{V}$ is lower semicontinuous at $V^{(\infty)} ;(2.4)$ then contradicts $(2.3)$ : consequently (2.2) is false.

Let now $\left\{V^{(r)}\right\}(b=1,2, \cdots)$ be a sequence of varieties of $\mathscr{F}$ such that

$$
I_{V}(r)<i+\frac{1}{r}
$$

By the compactness Theorem 2.b.2 there exists a variety $V^{(\infty)}$ of accumulation for $\left\{V^{(r)}\right\}$; semicontinuity Theorem 1.1 implies that $V^{(\infty)}$ is 
ordinary, because $I_{V}$ is upper bounded on $\left\{V^{(r)}\right\}$; hence, since $\mathscr{F}$ is a complete class, $V^{(\infty)} \in \mathscr{F}$. Semicontinuity Theorem 1.1 also yields

$$
I_{V(\infty)} \leqq \min \lim _{r \rightarrow \infty} I_{V}(r) ;
$$

consequently

$$
I_{V(\infty)}=i
$$

which completely proves Theorem 2.b.3.

Remark. Particular cases of the theorem of existence of the minimum have been proved previously, by Tonelli $[13,15,16$,] in the case $m=1, n=1$ (simple integrals) and $m=1, n=2$ (double integrals), and by this author [9] in the case $m=2, n=1$ (Fubini-Tonelli integrals). However this new general proof is considerably simpler than all those given in $[9,13,15,16]$.

(c) The concept of regular class needs discussion. In fact, this concept can only be accepted after proving that semicontinuous alphaasymptotic integrals may not have any minimum in some complete classes of ordinary varieties which are not regular. This can be shown by means of the following example (see also [9]):

Let $m=2, n=1, D_{1} \equiv D_{2} \equiv[0, \pi], \phi[x, y, p] \equiv\left(p_{1}^{1} \cdot p_{2}^{1}\right)^{2} . \quad I_{V}$ is lower semicontinuous and alpha asymptotic for any $\alpha \leqq 1$. Consider the sequence $V^{(r)},(r=1,2, \cdots)$ of varieties whose components are

$$
y_{1}(x)=\sin \left(r x_{1}\right) \text { and } y_{2}(x)=r^{-3} \sin \left(r x_{2}\right),
$$

respectively; $\left\{V^{(r)}\right\}$ is a complete class, because there are no varieties of accumulation. Each $V^{(r)}$ is ordinary, and

$$
I_{V}(r)=\int_{0}^{\pi} \int_{0}^{\pi} r^{-2} \cos ^{2}\left(r x_{1}\right) \cos ^{2}\left(r x_{2}\right) d x_{1} d x_{2}=\left(\frac{\pi}{r}\right)^{2} ;
$$

the lower bound of $I_{V}$ over $V^{(r)}$ is 0 , but $I_{V}$ never vanishes in $\left\{V^{(r)}\right\}$.

The fact that $\left\{V^{(r)}\right\}$ is not a regular class can also be shown directly without difficulty.

\section{BIBLIOGRAPHY}

1. S. Cinquini, Condizioni necessarie per la semicontinuitá degli integrali doppi del Calcolo delle Variazioni, Ann. Mat. Pura. Appl., 10, (1939) 233.

2. S. Faedo, Condizioni necessarie per la semicontinuitá di un nuovo tipo di funzionali, Ann. Mat. Pura. Appl., (4) 23 (1944) 69.

3. - Un nuovo tipo di funzionali continui, Rend. Mat. e. Appl., (5) 4 (1943) 1.

4. - Sulle condizioni di Legendre e di Weierstrass per gli integrali di Fubini-

Tonelli, Ann. Scuola Norm. Sup. Pisa, 15 (1950) 127. 
5. G. Fubini, Alcuni nuovi problemi di Calcolo delle Variazioni con applicazioni alla teoria delle equazioni integro-differenziali, Annali di Matematica, (3), 20 (1913) 217.

6. L. Giuliano, Sulle condizioni sufficienti per la semicontinuitá degli integrali doppi del Calcolo delle Variazioni, Ann. Scuola Norm. Sup. Pisa, 1941.

7. - Osservazioni sopra alcuni teoremi di semicontinuitá degli integrali doppi, Idem, 1914.

8. L. A. Lombardi, Sulla semicontinuitá degli integrali di Fubini-Tonelli, Ann. Scuola Norm. Super. Pisa, (3), 12 (1958) 129; MR 21 \#31, Zbl. 83, 327.

9. — Sull'esistenza del minimo degli integrali di Fubini-Tonelli, Rend. Ist. Lombardo Sci. 92 (1958) 446; MR 21 \#7460, Zb1. 84, 319.

10. - Sur la methode du gradient dans le Calcul des Variations, Comptes-Rendus du Congrés “Les Mathematiques de l'Ingenieur", Mons-Bruxelles (1958); Zbl. 92, 107.

11. - The continuity of the most general integral of the Calculus of variations in non-parametric form, J. of Math. Analysis and Applications, (2) 3 (1961), 335-350.

12. - The semicontinuity of the most general integral of the calculus of variations in non-parametric form, Pacific J. Math., (11) 4 (1961), 1407-1420.

13. L. Tonelli, Fondamenti di Calcolo delle Variazioni, Vols. I and II, Zanichelli, Bologna, (1921).

14. - Sur la sémicontinuité des integrales doubles du Calcul des Variations, Acta Math. 53 (1939) 323.

15. - Gli integrali del Calcolo delle Variazioni in forma ordinaria, Ann. Scuolla Norm. Super. Pisa, (2), 3, 401.

16. - L'estremo assoluto degli integrali doppi, Ann. Scuola Norm. Super., (2), 2 (1933) 89.

17. - Sull'appossimazione analitica delle funzioni di piú variabili, Rend. Circ. Mat. Palermo, (1910).

18. — - Su alcuni funzionali, Annali di Matematica (4), 18 (1939).

UNIVERSity of CALIFornia at Los ANGEles 



\title{
PACIFIC JOURNAL OF MATHEMATICS
}

\author{
EDITORS
}

Ralph S. Phillips

Stanford University

Stanford, California

M. G. Arsove

University of Washington

Seattle 5, Washington
A. L. Whiteman

University of Southern California Los Angeles 7, California

Lowell J. Paige

University of California

Los Angeles 24, California

\section{ASSOCIATE EDITORS}
E. F. BECKENBACH
D. DERRY
M. OHTSUKA
H. L. ROYDEN
E. SPANIER
E. G. STRAUS
T. M. CHERRY
F. WOLF

\section{SUPPORTING INSTITUTIONS}

\author{
UNIVERSITY OF BRITISH COLUMBIA \\ CALIFORNIA INSTITUTE OF TECHNOLOGY \\ UNIVERSITY OF CALIFORNIA \\ MONTANA STATE UNIVERSITY \\ UNIVERSITY OF NEVADA \\ NEW MEXICO STATE UNIVERSITY \\ OREGON STATE UNIVERSITY \\ UNIVERSITY OF OREGON \\ OSAKA UNIVERSITY \\ UNIVERSITY OF SOUTHERN CALIFORNIA
}

\author{
STANFORD UNIVERSITY \\ UNIVERSITY OF TOKYO \\ UNIVERSITY OF UTAH \\ WASHINGTON STATE UNIVERSITY \\ UNIVERSITY OF WASHINGTON \\ $*$
AMERICAN MATHEMATICAL SOCIETY \\ CALIFORNIA RESEARCH CORPORATION \\ SPACE TECHNOLOGY LABORATORIES \\ NAVAL ORDNANCE TEST STATION
}

Mathematical papers intended for publication in the Pacific Journal of Mathematics should be typewritten (double spaced), and the author should keep a complete copy. Manuscripts may be sent to any one of the four editors. All other communications to the editors should be addressed to the managing editor, L. J. Paige at the University of California, Los Angeles 24, California.

50 reprints per author of each article are furnished free of charge; additional copies may be obtained at cost in multiples of 50 .

The Pacific Journal of Mathematics is published quarterly, in March, June, September, and December. Effective with Volume 13 the price per volume (4 numbers) is $\$ 18.00$; single issues, $\$ 5.00$. Special price for current issues to individual faculty members of supporting institutions and to individual members of the American Mathematical Society: $\$ 8.00$ per volume; single issues $\$ 2.50$. Back numbers are available.

Subscriptions, orders for back numbers, and changes of address should be sent to Pacific Journal of Mathematics, 103 Highland Boulevard, Berkeley 8, California.

Printed at Kokusai Bunken Insatsusha (International Academic Printing Co., Ltd.), No. 6, 2-chome, Fujimi-cho, Chiyoda-ku, Tokyo, Japan.

PUBLISHED BY PACIFIC JOURNAL OF MATHEMATICS, A NON-PROFIT CORPORATION

The Supporting Institutions listed above contribute to the cost of publication of this Journal, but they are not owners or publishers and have no responsibility for its content or policies. 


\section{Pacific Journal of Mathematics}

\section{Vol. 12, No. $2 \quad$ February, 1962}

William George Bade and Robert S. Freeman, Closed extensions of the Laplace operator determined by a general class of boundary conditions . . . . . . . 395

William Browder and Edwin Spanier, H-spaces and duality ............. 411

Stewart S. Cairns, On permutations induced by linear value functions . . . . . . . 415

Frank Sydney Cater, On Hilbert space operators and operator roots of

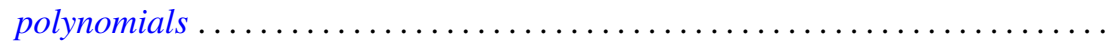

Stephen Urban Chase, Torsion-free modules over $K[x, y] \ldots \ldots \ldots \ldots \ldots \ldots \ldots 437$

Heron S. Collins, Remarks on affine semigroups . . . . . . . . . . . . . . . . 449

Peter Crawley, Direct decompositions with finite dimensional factors . . . . . . . 457

Richard Brian Darst, A continuity property for vector valued measurable

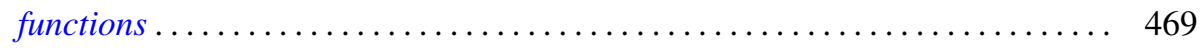

R. P. Dilworth, Abstract commutative ideal theory ................. 481

P. H. Doyle, III and John Gilbert Hocking, Continuously invertible spaces . . . . . . 499

Shaul Foguel, Markov processes with stationary measure . . . . . . . . . . . 505

Andrew Mattei Gleason, The abstract theorem of Cauchy-Weil ............ 511

Allan Brasted Gray, Jr., Normal subgroups of monomial groups . . . . . . . . . . 527

Melvin Henriksen and John Rolfe Isbell, Lattice-ordered rings and function

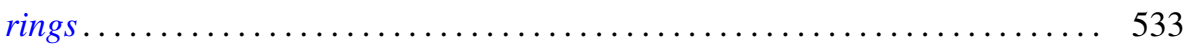

Amnon Jakimovski, Tauberian constants for the $[J, f(x)]$ transformations . ..... 567

Hubert Collings Kennedy, Group membership in semigroups . . . . . . . . . . . 577

Eleanor Killam, The spectrum and the radical in locally $m$-convex algebras ..... 581

Arthur H. Kruse, Completion of mathematical systems . . . . . . . . . . . . . 589

Magnus Lindberg, On two Tauberian remainder theorems ................ 607

Lionello A. Lombardi, A general solution of Tonelli's problem of the calculus of

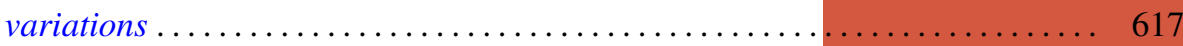

Marvin David Marcus and Morris Newman, The sum of the elements of the powers

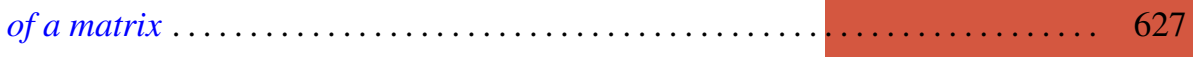

Michael Bahir Maschler, Derivatives of the harmonic measures in

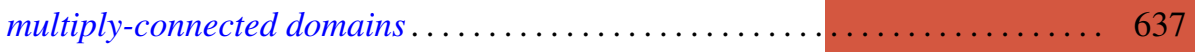

Deane Montgomery and Hans Samelson, On the action of $\mathrm{SO}(3)$ on $S^{n} \ldots \ldots \ldots 649$

J. Barros-Neto, Analytic composition kernels on Lie groups . . . . . . . . . . . . 661

Mario Petrich, Semicharacters of the Cartesian product of two semigroups ...... 679

John Sydney Pym, Idempotent measures on semigroups . . . . . . . . . . . . 685

K. Rogers and Ernst Gabor Straus, A special class of matrices . . . . . . . . . . . . 699

U. Shukla, On the projective cover of a module and related results . . . . . . . . . 709

Don Harrell Tucker, An existence theorem for a Goursat problem . . . . . . . . . . . 719

George Gustave Weill, Reproducing kernels and orthogonal kernels for analytic

differentials on Riemann surfaces ......................... 729

George Gustave Weill, Capacity differentials on open Riemann surfaces ........ 769

G. K. White, Iterations of generalized Euler functions . . . . . . . . . . . . . 777

Adil Mohamed Yaqub, On certain finite rings and ring-logics . . . . . . . . . 785 